\title{
TV/Series
}

$1 \mid 2012$

Les Séries télévisées américaines contemporaines :

entre la fiction, les faits, et le réel

\section{Patty, Vic, Jack et les autres : antihéros modernes et postmodernes dans les séries américaines contemporaines}

\section{Alexis Pichard}

\section{OpenEdition}

\section{Journals}

Édition électronique

URL : http://journals.openedition.org/tvseries/1574

DOI : 10.4000/tvseries.1574

ISSN : 2266-0909

Éditeur

GRIC - Groupe de recherche Identités et Cultures

\section{Référence électronique}

Alexis Pichard, «Patty, Vic, Jack et les autres : antihéros modernes et postmodernes dans les séries américaines contemporaines ", TV/Series [En ligne], 1 | 2012, mis en ligne le 15 mai 2012, consulté le 20 avril 2019. URL : http://journals.openedition.org/tvseries/1574 ; DOI : 10.4000/tvseries.1574

\section{(c) (i) (9)}

TV/Series est mis à disposition selon les termes de la licence Creative Commons Attribution - Pas d'Utilisation Commerciale - Pas de Modification 4.0 International. 
Patty, Vic, Jack et les autres : antihéros modernes et postmodernes dans les séries américaines contemporaines.
« Ils ne sont pas aimables, et pourtant, ils nous fascinent, nous les comprenons à des niveaux très profonds et nous excusons leur comportement [...]. Il faut juste espérer que le public a assez d'éléments pour comprendre, ou du moins rationaliser, la conduite de votre personnage et pour voir ce qui le gouverne ${ }^{1} »$. Jon Hamm.

$\mathrm{J}$

on Hamm, interprète de Don Draper dans la série Mad Men (AMC, 2007-), donne ici une définition assez juste de la plupart des protagonistes présents dans les séries américaines contemporaines. Peu aimables, parfois détestables, ils s'éloigneraient des conceptions traditionnelles du héros. Pour autant, le public ne semble pas s'en détourner et apparait même comme fasciné, allant jusqu'à excuser des actes odieux dans la mesure où la série les légitime. C'est d'évidence le cas de Don Draper, personnage dont la duplicité est peu à peu «expliquée » par les stigmates laissés par une enfance douloureuse. Don Draper n'est ni hérö̈que, ni foncièrement maléfique - son ambivalence nous invite à le considérer davantage comme un antihéros dont il est loin d'être le seul représentant. De nombreuses séries marquantes des années 2000 comme The Shield (F/X, 20022009), House MD (Dr House, Fox, 2003-) ou Damages (F/X, 20072010 ; DirecTV, 2011-2012), mettent ainsi en avant un genre de protagoniste corrompu, cynique ou débauché qui tend à remplacer la figure du héros triomphant. Se pose alors la question de savoir pourquoi et comment ces protagonistes longtemps restés minoritaires sur le petit écran sont devenus à ce point populaires durant les années 2000

${ }^{1}$ Interview de Jon Hamm in Séries TV \#46, avril/mai 2010. 
Si, dès les années 1960 , la télévision nous offre de manière sporadique quelques cas d'antihéros, ces derniers vont connaître une véritable floraison durant les années 2000 , à l'heure où le réalisme devient un argument constitutif des nouvelles séries. L'étude de cette récurrence nous permettra d'une part, d'envisager une définition de l'antihéros à l'aune des productions contemporaines, et d'autre part, d'en dresser une typologie. L'ensemble de cette démarche nous invitera ainsi à porter un regard global et critique sur ceux qui ont contribué à bouleverser le paysage télévisuel en lui insufflant noirceur et réalisme.

\section{Le triomphe des antihéros.}

La figure de l'antihéros n'est pas récente puisqu'on la trouve déjà dans la littérature du début du XVIIe siècle avec par exemple le personnage de Don Quichotte créé par Cervantès. Après avoir été repris par le cinéma (notamment dans Le Gaucher ou plus tard dans L'inspecteur $\mathrm{Harry}^{2}$ ), l'antihéros est présent à la télévision dès la fin des années 60 avec l'apparition notamment de Columbo (NBC, 19682003). Grâce à ses méthodes peu orthodoxes et sa dégaine singulière qui met à mal le prestige du détective traditionnel, le lieutenant Columbo parvient toujours à résoudre ses enquêtes. C'est dans cette veine policière que s'inscrit Hill Street Blues (NBC, 1981-1987), série phare et très réaliste des années 1980 qui suit la vie d'un commissariat peuplé de policiers hauts en couleur. En 1993, son créateur, Steven Bochco, déjà scénariste de nombreux épisodes de Columbo, produit NYPD Blue (NBC, 1993-2005), nouvelle série policière portée par Sipovicz, inspecteur tourmenté ayant un penchant pour l'alcool qui préfigure la tendance des antihéros modernes à la télévision. Malgré tout, le public ne semble pas adhérer facilement à ce genre de personnages. Car si NYPD Blue est un succès, il n'en est pas de même pour la très brève mais audacieuse Profit (Fox, 1996) que le réseau Fox diffuse pendant seulement quatre semaines.

La série créée par le tandem MacNamara-Greenwalt était d'une grande noirceur et proposait un antihéros atypique car foncièrement malfaisant. Jim Profit, pur produit du capitalisme déraisonné des années 1980, est le Vice Président de la société Gracen\&Gracen. Il a grandi dans un carton estampillé du nom de cette même entreprise, abreuvé par le flux d'images émis par un poste de télévision allumé en continu. Vil, manipulateur, sournois, Profit apparaît comme le diable incarné dans une période où devenir riche était une devise collective, une ambition à la portée de tous (en théorie). A travers la narration en voix off du protagoniste, la série tente pourtant de créer une connivence entre lui et le téléspectateur. Mais comment s'identifier à un monstre? L'échec de Profit a sans

${ }^{2}$ Réalisés respectivement par Arthur Penn, 1958, et DonSiegel, 1971. 
doute montré que les téléspectateurs n'étaient pas encore prêts à se laisser entraîner dans l'esprit d'un sociopathe ou d'un personnage malveillant. Peut-être la série était-elle en avance sur son temps puisque trois ans plus tard, la chaîne câblée HBO lançait Les Soprano (HBO, 1999-2007), œuvre marquante de la fin de siècle qui devint le jalon fondateur de toute la fiction sérielle à venir.

En effet, en présentant Tony Soprano, parrain de la mafia du New Jersey, sous les traits d'un dépressif en thérapie, David Chase allait marquer l'histoire des séries américaines. Non seulement il centrait sa série sur un criminel implacable, mais il réussissait aussi à nous le rendre sympathique en développant les aléas de sa vie de famille, notamment ses relations difficiles avec sa mère, Livia, vieille femme aussi geignarde que manipulatrice. Le succès de la série fut exceptionnel : jusqu'à 13 millions de téléspectateurs la regardèrent sur HBO. La voie était à présent ouverte pour une nouvelle génération d'antihéros qui seraient des descendants plus ou moins conscients de Tony Soprano.

A la base, la volonté du câble de diffuser des séries centrées sur des antihéros était motivée par le désir de se démarquer des networks traditionnels qui continuaient à proposer une majorité de séries incorporant des héros manichéens. C'est en partie grâce à cette politique que $\mathrm{HBO}$ est très vite parvenue à se forger une image de chaîne novatrice et audacieuse. Plus sombres et ambigus, les antihéros de HBO inspirèrent aussi bien les autres chaînes du câble, FX en tête, que les réseaux, dans une moindre mesure. Ces derniers sont en effet soumis au contrôle de la Federal Communications Commission (FCC), équivalent de notre CSA. Leur degré de noirceur et de violence est donc plus limité. C'est ainsi qu'apparurent Vic Mackey (The Shield), Dexter Morgan (Dexter, Showtime, 2006-) ou encore Lucy Spiller (Dirt, F/X, 2007-2008). Les antihéros ont une personnalité complexe que le téléspectateur ne pourra décrypter que sur une période longue. Mais les années 2000 mettent aussi en exergue la diversité de modèles d'antihéros.

Cette déferlante d'antihéros s'explique également par le contexte politique durant lequel elle se produit. En septembre 2001, les attentats du Wall Trade Center font basculer les États-Unis dans la peur et la paranoïa. Marqués par la violence de cette attaque terroriste sans précédent, les Américains prennent soudain conscience de la fragilité de leur pays. L'armée et les agences gouvernementales (la CIA en tête) montrent leurs limites : elles ne parviennent pas à prévenir de manière efficace la menace terroriste. L'archétype du «bon » héros se brise, n'étant plus apte à protéger la nation. Si les terroristes n'ont pas d'honneur et sont capables de tuer des milliers d'innocents, au nom de quoi les Américains devraient-ils se préoccuper de respecter la loi ? En d'autres termes, puisque les terroristes ont dépassé les limites, l'Amérique doit répondre avec la même violence. La menace doit être 
contrée, coûte que coûte. Un tel argumentaire légitime l'action d'un antihéros comme Jack Bauer qui est la parfaite incarnation de l'après 11 septembre. La série télévisée apparaît ici comme le reflet de l'inconscient collectif et joue exactement le rôle qu'a décrit Bettelheim à propos des contes de fées. En mettant en images les pires scénarios possibles et en montrant les méthodes utilisées pour les éviter, elle répond aux angoisses de chacun ${ }^{3}$.

Par ailleurs, cette vague d'antihéros survient alors que la téléréalité est devenue le genre télévisuel le plus populaire avec des émissions comme Survivor ou American Idol. Le public se passionne pour ces programmes qui prétendent filmer le réel, des candidats issus de la vie de tous les jours. Les héros ne sont plus des surhommes mais bien des personnes ordinaires. En découle une identification spectatorielle amplifiée. Comme le public, les candidats des émissions de téléréalité ont des problèmes que les animateurs promettent de résoudre, que ce soit une rupture délicate (Breaking up with Shannen Doherty), des problèmes dans l'éducation des enfants (Supernanny) ou des difficultés à s'accepter (Extreme Makeover). En outre, ces inconnus ressemblent aux téléspectateurs. Leurs failles et leurs faiblesses les rendent d'autant plus humains et attachants. Tout cela, les producteurs de séries télévisées le comprennent rapidement. Les héros comme figures de perfection ne répondent plus aux attentes du public qui veut davantage de réel, de vraisemblable, des personnages fragiles, ordinaires, qui ont des défauts. Les antihéros apparaissent comme une réponse adaptée. Pour faciliter et rendre encore plus forte l'identification du téléspectateur, les scénaristes n'hésitent pas à pénétrer dans la vie intime des personnages. Ainsi, voir Tony Soprano, mafieux craint par tous, évoquer sa relation avec sa mère au cours d'une séance de thérapie, forge une connivence entre lui et le téléspectateur car nous découvrons son humanité au travers de sa fragilité. Quant à Lucy Spiller, rédactrice en chef machiavélique d'un journal à scandales, c'est la découverte progressive de son passé et de sa vie personnelle tourmentés ${ }^{4}$ qui la rend humaine. Il en va de même pour Dexter Morgan : la sympathie que nous éprouvons à l'égard du personnage n'est pas uniquement due à son terrible passé. La voix-off de Dexter qui accompagne les épisodes joue également beaucoup dans notre identification compassionnelle. Ses réflexions teintées d'incompréhension sur le fonctionnement des émotions, qu'il n'est pas capable de ressentir, nous offrent un accès privilégié à son psychisme. Comme le justifie Michael C. Hall, l'interprète de Dexter : « la voix-off

\footnotetext{
3 Bruno Bettelheim, Psychologie des contes de fées, Paris, Pocket, 1999.

${ }^{4}$ Le suicide de son père lorsqu'elle n'avait que quinze ans la hante d'autant plus que celui-ci lui a laissé un mot des plus troublants.
} 
[...] plonge le spectateur dans une intimité du héros qu'aucun personnage de la série ne partage. Vous faites partie du voyage $» 5$.

Tous ces procédés tendent à montrer que ces antihéros sont humains. Si le héros est d'une nature différente et se situe dans une autre dimension, au-delà des mortels, l'antihéros quant à lui, même s'il est doté de qualités exceptionnelles (plus performant, plus courageux, plus fort, plus malin etc.) reste accessible : il est sur le même plan que chacun d'entre nous et place son héroïsme à la portée de tous. Il est certes supérieur mais demeure humain. C'est précisément cette démocratisation, cet " héroïsme du même » selon l'expression d'A. Ehrenberg6, qui va favoriser l'identification à l'antihéros. Ce phénomène est particulièrement évident chez les « antihéros malgré eux $\gg 7$ car ils sont souvent l'archétype du commun des mortels. Par exemple, quand Chuck Bartowsky (Chuck, NBC, 2007-2012), simple informaticien dans un magasin d'électroménager local, devient agent secret par un concours de circonstances, nous éprouvons de la compassion à son égard car il n'a aucun des pré-requis pour le devenir.

Enfin, on note un dernier élément d'explication pour comprendre la récente omniprésence des antihéros : la première partie des années 2000 a fait la part belle aux séries feuilletonesques avec des arcs narratifs s'étendant parfois sur des saisons entières. En revanche, depuis 2007, c'est le formula show policier qui réinvestit les écrans avec des intrigues s'éloignant de la logique du feuilleton, développées et bouclées dans un seul épisode. Cette tendance est flagrante sur les networks qui considèrent à présent les séries davantage comme un produit au court terme, épisode après épisode, par souci d'obtenir un succès immédiat. Malgré tout, les scénaristes continuent d'injecter une part de feuilletonesque. La psychologie de personnages complexes, le plus souvent des antihéros, devient alors le fil rouge qui va tenir le téléspectateur en haleine. C'est par exemple tout le sel d'une série comme House MD. Même si l'intrigue nous intéresse, c'est bien l'acariâtre Gregory House qui est le pilier, l'intérêt majeur de la série : le téléspectateur va peu à peu apprendre à le connaître et ainsi comprendre la raison de sa misanthropie. Les titres des séries de ce genre l'illustrent bien - à la manière de Monk (USA Network, 20022009) ou Nurse Jackie (Showtime, 2009-) - c'est l'antihéros qui fait leur identité.

\section{Définir l'antihéros à l'aune des séries contemporaines}

\footnotetext{
5 Interview de Michael C.Hall in Séries TV\#48, octobre/novembre 2008.

${ }^{6}$ Alain Ehrenberg, Le culte de la performance, Paris, Calmann-Lévy, 1991.

7 Voir troisième partie, «Une typologie des antihéros modernes dans les séries dramatiques », p 11.
} 
Pour bien cerner cette notion d'antihéros, particulièrement malmenée dans les média ${ }^{8}$, il faut d'abord revenir à la figure de référence : le héros. Dans la Grèce antique, le "héros » désigne les demi-dieux et les grands hommes divinisés, des personnages légendaires aux qualités et aux exploits extraordinaires, dotés d'un très grand courage et qui font preuve d'une vertu exceptionnelle. Le héros est par ailleurs le personnage central d'une œuvre de fiction, aussi appelé "protagoniste ». C'est bien l'union de ces caractéristiques qui est intéressante lorsque l'on tente de définir ce qu'est un antihéros. Dans antihéros, se trouve bien la notion de « héros ». A trop vouloir se focaliser sur le préfixe « anti », l'antihéros tend bien souvent à être assimilé à un être forcément vil, amoral, antipathique ou encore pervers. Or, il semble que dans la majorité des cas, l'antihéros ne se limite pas à cette définition et répond à plusieurs impératifs qu'on retrouve d'une série à une autre.

L'antihéros est bien le personnage central d'une œuvre, le protagoniste, mais, contrairement au héros traditionnel, il n'est pas seulement constitué de qualités et apparaît, de fait, moins monolithique. Pourtant, comme le héros, il s'affirme comme étant guidé par une éthique, par une perspective téléologique. Les antihéros visent tous ce « souverain bien ${ }^{9}$ » que Kant définit comme l'accession au bonheur. La vertu (la volonté du bien) étant la condition du bonheur (bien absolu), le « souverain bien » est un bonheur moral. Mais, ce qui différencie l'antihéros du Héros, c'est précisément son rapport spécifique au concept de Kant. Jack Bauer, dans 24 heures chrono rêve de sauver l'Amérique. Vic MacKey, le policier de The Shield cherche à éradiquer la délinquance dans Farmington, quartier fictif et mal famé de Los Angeles. Patty Hewes, quant à elle, aspire, dans la série Damages, à protéger les «petits citoyens » contre les grands prédateurs de la finance. Enfin, Dexter Morgan se place en justicier en empêchant des meurtriers relaxés de commettre un nouveau crime. Néanmoins, le recours à la torture, à la corruption, au mensonge et même au meurtre apparaissent comme autant de moyens qui ne sont ni déontologiques, ni moraux. Ces personnages aspirent à accomplir leur devoir à finalité morale mais ont recours à des moyens qui défient la morale. Leur comportement est ainsi clairement anomique ${ }^{10}$ dans la mesure où, s'ils semblent vouloir se conformer aux idéaux culturels valorisés par la société, les antihéros utilisent des méthodes pour les

\footnotetext{
${ }^{8}$ Ainsi, dans son article intitulé « Autopsie du antihéros » (excessif.com, 18 février 2010), Geoffrey Crête donne une vision univoque de l'antihéros : "l'antihéros est donc amoral, antipathique, cupide, cynique, pervers, névrosé, voire idiot et pathétique.» Or, nous verrons que c'est précisément l'ambivalence qui caractérise ce genre de personnages.

Emmanuel Kant, Fondements de la mécanique des mours, Paris, Delagrave, 1999.

${ }^{10}$ Selon R.K.Merton (Social Theory and Social Structure, New York, Macmillan, 1949), l'anomie correspond à un comportement qui, tout en acceptant les objectifs culturels, ne respecte pas les normes sociales préconisées.
} 
atteindre qui sont contraires aux usages. Ce qui génère un personnage qui semble finalement assez peu se différencier du «bad guy » et qui peut quelquefois laisser croire que l'antihéros est un antagoniste, le parfait pendant maléfique du héros. Le trouble est jeté et comme dirait Nathan Ford de la série Leverage (TNT, 2008-) : « and sometimes, bad guys make the best good guys ${ }^{11}$ (4.08). On ne peut d'ailleurs manquer de souligner que ces passages réitérés du «côté obscur » constituent un atout exclusif et décisif de ces protagonistes en leur donnant une connaissance très claire de ce qu'est le Mal. Car l'une des limites du héros, c'est précisément son incapacité à le comprendre. Jack Bauer, Dexter Morgan et Vic Mackey possèdent ainsi cet avantage qui les rend si efficaces : ils sont d'autant plus aptes à avoir un temps d'avance que les réactions des « méchants » ne leur sont pas inconnues.

Apparaissant donc souvent à la fois comme le « gentil méchant » et le " méchant gentil », l'antihéros incarne, en définitive, la résolution dialectique de l'opposition entre le Bien et le Mal. Car Vic Mackey ne fait pas le Bien à certains moments et, à d'autres, le Mal; Dexter n'est pas bon aujourd'hui, mauvais demain. Ils dépassent ces oppositions en se situant au-delà du Bien et du Mal. Ainsi, Patty Hewes fait preuve d'altruisme par pur calcul égoïste, Jack Bauer a recours à la torture pour sauver des milliers de vies, et Dexter rend la justice en assassinant des tueurs récidivistes. Les antihéros évoluent dans un maelstrom qui les entraine irrésistiblement à dépasser des termes moraux qui ne sont ni univoques, ni intangibles. Dans leur réalité, la morale n'est qu'une illusion qui ne leur est d'aucun secours dans ces situations paroxystiques où les conflits de devoirs sont tels qu'il n'existe plus aucune règle pour indiquer le bon chemin. C'est ainsi que, tenaillé entre l'impératif de sauver la Nation et son devoir de loyauté familiale, Jack Bauer doit faire un choix à la fin de la $5^{\text {ème }}$ heure de la

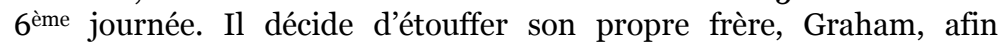
d'obtenir des renseignements sur un terroriste présumé, en l'occurrence son père. Dexter Morgan sera, quant à lui, confronté à un dilemme encore plus pénible lorsque, pour sauver sa sœur Debra, il n'aura d'autre solution que de tuer son frère Rudy (1.12) même si, dans ce cas, la psychopathie de ce dernier facilite la décision. De même, confronté au conflit entre le devoir de proposer le meilleur traitement à un patient et l'impératif de respecter les choix du malade, Dr House choisit d'intuber John Henry Giles, un patient qui avait pourtant refusé (1.9) et clôt le débat par un sarcastique : « to intubate or not, that is the big ethical question ${ }^{12}$ ». Dans ces instants où la morale atteint ses limites, les antihéros ne sont plus guidés que par leur intuition ou leur conviction.

\footnotetext{
11 « Parfois, ce sont les méchants qui font les meilleurs gentils »

12 «Intuber ou ne pas intuber, telle est la grande question éthique »
} 
Ainsi, nous approchons d'une définition qui rassemblerait une grande partie de nos personnages. L'antihéros serait tout simplement un héros désabusé qui, tout en continuant à rêver d'atteindre un «souverain bien » dont l'image se brouille, est conduit, par sa réalité quotidienne, vers une éthique fondamentalement amorale. ${ }^{13}$ Tous ces personnages entretiennent l'illusion de faire le Bien mais ils semblent aussi conscients que leur réalité les mène à transcender les frontières entre les valeurs morales. Confrontés à la réalité, ils agissent avec une sorte de «sagesse pratique », une sagacité qu'évoquait Aristote sous le nom de phronesis ${ }^{14}$, autrement dit cette faculté de faire des choix dans des circonstances concrètes, chaque fois différentes et pour une part imprévisibles, sans forcément tenir compte des principes moraux universels.

On comprend bien que l'antihéros ne saurait être parfait. Bien sûr c'est un héros, il finit donc toujours par réussir mais au prix d'une série de ratages et d'échecs qui engendrent des dégâts collatéraux très lourds. Ainsi, dans 24, on ne compte plus les épisodes où Bauer arrive trop tard, par exemple, après qu'une bombe a explosé au beau milieu d'un centre commercial. Quant à Dexter Morgan, en dépit de son code qui lui dicte de ne tuer que des coupables, il en arrive à se tromper et à exécuter un innocent photographe (4.7). Même le Docteur House ne parvient pas à sauver tous ses patients. Par ailleurs, l'antihéros apparaît comme la figure d'un paradoxe : l'efficacité dont il fait preuve pour accomplir sa mission contraste avec le chaos qui régit sa vie personnelle. S’il finit par réussir à venir à bout de sa tâche, cela se fait le plus souvent au détriment de sa vie familiale ou sentimentale. Ainsi, aucun des protagonistes mentionnés précédemment n'a pu conserver le moindre conjoint. Mackey et Hewes ont divorcé, et les épouses de Bauer et Morgan ont été assassinées. Les relations qu'ils entretiennent avec leurs enfants sont tout aussi conflictuelles pour la grande majorité d'entre eux. La fille de Jack Bauer refuse de le revoir pendant plusieurs journées, celle de Vic Mackey finit par s'enfuir et le fils de Patty Hewes la déteste à tel point qu'il essaiera de la tuer lors de la troisième saison. Quant à leurs frères et sœurs, les antihéros finissent par les torturer (pour Bauer dans la saison 2), les laisser en prison (pour Ellen Parson, protagoniste de Damages dans la saison 3), ou encore les tuer (pour Dexter dans l'épilogue de la saison 1). Un sort similaire est parfois réservé à leurs parents à l'image de Philip Bauer que son fils Jack fait exploser sur une plate-forme off-shore à la fin de la 6 ème journée.

L'antihéros des séries télévisées possède un attribut supplémentaire: il se transforme au fur et à mesure de son cheminement. La structure du récit sériel feuilletonnesque,

${ }^{13}$ La morale est constituée des règles qui dirigent nos actions (comment vivre ?) ; elle traduit en actes l'exigence éthique qui propose les grandes directions (que dois-je faire ?).

${ }^{14}$ Aristote, Ethique à Nicomaque, Garnier-Flammarion, 1965 (livre VI, 8). 
développant une histoire sur plusieurs saisons, permet d'enrichir sa psychologie. La multiplication des personnages qui approchent le protagoniste sous différents angles, les flashbacks, le développement des intrigues personnelles autour de l'antihéros, sont autant d'occasions d'arpenter les méandres de leur psychologie tourmentée. D'autant que, au moins pour les séries à succès, la réalisation de plusieurs saisons se déroulant à plusieurs années d'intervalle permet le plus souvent de faire évoluer le caractère du protagoniste d'une façon considérable. C'est le cas de Dexter Morgan, psychopathe ne ressentant aucune émotion dans sa première saison, qui s'humanise peu à peu au contact de l'amour de ses proches. Ce processus sera néanmoins bouleversé par la mort tragique de plusieurs de ces figures aimantes et Dexter retrouvera alors sa nature profonde la plus sombre. Néanmoins, le Dexter qui émerge à l'issue de la saison 5 n'a plus grand-chose à voir avec ses débuts de serial killer traditionnel. L'évolution de Jack Bauer est tout aussi intéressante dans la mesure où la durée de la série va permettre de soumettre sa psychologie aux changements du climat sociopolitique dans lequel il évolue. Il a souvent été dit que Bauer était l'incarnation du héros bushien par excellence, avec ses réminiscences d'idéologie conservatrice, sa légitimation de la torture (67 actes de torture recensés durant les 5 premières journées ${ }^{15}$ ) ou encore son obsession de l'ennemi étranger. Si l'on peut débattre sur le caractère apologique de la série, il s'avère néanmoins que Bauer se pose peu de questions existentielles durant les premières saisons. En revanche, le héros qui nous est proposé à partir de la saison 5 semble préfigurer l'Amérique d'Obama. C'est l'heure des bilans et des doutes, le temps des remises en question qui nous montre un héros drogué, détruit, simple machine à tuer ayant perdu toute illusion et dont la visée éthique semble évanouie. Le travail doit être bien fait mais est-il encore bien de le faire? Les dernières heures de la 8ème journée de Jack Bauer seront révélatrices, le montrant entièrement emporté par un souci de vengeance personnelle qui le mène à une violence qui n'a plus rien de fondatrice.

Dans certains cas pourtant, le caractère feuilletonnesque permet au scénariste de laisser croire que l'antihéros évolue alors qu'au final, il aura conservé les mêmes traits de caractères. L'exemple de Vic

15 Dans son article intitulé "Jack's back: the clock ticks for 24's antihero" (www.independent.co.uk, 21 novembre 2008), Robert Hanks précise à ce sujet : " During the first five seasons, according to the Parents Television Council in the US, 24 depicted 67 acts of torture. Over 120 episodes, that's just over once every two shows. Put another way, it suggests that Jack Bauer personally tortures somebody once every three to four hours, which is hardly a healthy lifestyle. 
Mackey est à cet égard pertinent. On peut en effet arguer qu'il fait partie de ces rares antihéros à ne pas évoluer. Et ce, même si une fois le crime originel passé, on se surprend à éprouver des sentiments plus positifs envers lui lorsqu'on le découvre en père ou mari protecteur, en collègue ayant le respect de son équipe ou plus simplement en agent de terrain cherchant à faire baisser la criminalité. Autant de détails qui peuvent laisser penser que Vic évolue lentement, et qui nous font oublier l'horreur de son crime. La leçon de Profit a bien été retenue : on peut avoir un antihéros très sombre, mais le montrer constamment sous ce seul angle reste peu favorable à l'adhésion du téléspectateur. Pour pouvoir s'en rapprocher, ce dernier doit voir son personnage quitter ses habits de méchant avec le temps.

$\mathrm{Au}$ final, si la figure supra-humaine du Héros est bel et bien sacrée au sens premier (du latin sacer, séparer), celle de l'antihéros est bien plus riche et épuise toute la polysémie de ce terme. A la fois être à part, différent du commun de ses congénères par des qualités exceptionnelles, il est également celui qui est maudit (homo sacer) comme le secrétaire de la Défense Heller l'assène à Jack Bauer (6.20): "You're cursed Jack. Everything you touch, one way or another, ends up dead ${ }^{16}$. » De fait, au-delà même de celui qui se confond avec elle, tous les antihéros semblent avoir leur part de malédiction. Ainsi Vic Mackey, à qui la phrase d'Heller aurait tout aussi bien convenu, paraît véritablement victime d'une malédiction, ne serait-ce qu'avec Chasity et Matt, ses deux enfants autistes. Assurant le «sale boulot», l'antihéros peut également être désigné comme «bouc émissaire » et sacrifié (sacer facere) sur l'autel de la raison d'Etat comme c'est le cas de Bauer lors de la $1^{\text {ère }}$ heure de la 6 ème journée : après l'avoir laissé dépérir dans les geôles chinoises pendant vingt mois, le Président Wayne Palmer le livre à Abu Fayed pour endiguer une vague d'attentats terroristes aux États-Unis. Jack Bauer est d'ailleurs souvent abandonné par sa hiérarchie et ses actions seront même directement

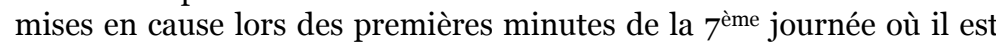
désigné comme le responsable d'un type de contre-espionnage peu respectueux des droits de l'homme.

\section{Une typologie des antihéros dans les séries télévisées contemporaines.}

La pluralité des antihéros dans les années 2000 invite à nous intéresser aussi bien à ce qui les rassemble qu'à ce qui les oppose. Afin de les cerner au mieux, nous nous proposons d'en établir une typologie qui, sans prétendre à l'exhaustivité, permet de classer la plus grande

${ }^{16}$ «Vous êtes maudit, Jack. Tout ce que vous touchez, d'une manière ou d'une autre, finit par mourir. » 
partie d'entre eux en trois catégories majeures que sont les antihéros malgré eux, les machiavéliques et les maudits.

\section{L'antihéros malgré lui.}

Lorsque l'on essaie de définir l'antihéros malgré lui, on pense généralement à un héros qui ne correspond pas aux attentes traditionnelles. Dans les années soixante, l'un des premiers avatars du genre apparaît en la personne de Maxwell Smart, héros de la série Get Smart (Max la Menace, NBC, 1965-1969, puis CBS, 1969-1970). Cet agent secret se démarque par son incompétence qui va immanquablement mettre en péril ses missions. Il s'éloigne en tout point de l'idée que l'on se fait de l'agent secret. Pourtant, même s'il n'est ni beau, ni musclé, ni très intelligent, il va souvent miraculeusement déjouer les plans du KAOS, organisation internationale diabolique. Cela donne lieu à des séquences cocasses où le protagoniste en viendrait presque à s'excuser de sa maladresse qui va contre toute attente se révéler salvatrice. De plus, l'humour qui soustend les tribulations de ce genre d'antihéros est aussi lié à un parcours initiatique laborieux. Ces antihéros ne sont pas préparés au destin héroïque qui s'abat sur eux et qu'ils vont porter comme un fardeau. Ils doivent donc apprendre à devenir ce qu'ils ne sont pas : des héros.

Reprenant sensiblement les mêmes codes que Get Smart, la série d'espionnage drolatique Chuck se révèle une parfaite illustration des antihéros malgré eux durant les années 2000. Dans cette dramatique, Chuck Bartowky, un geek travaillant dans un magasin d'électroménager, devient du jour au lendemain le meilleur atout des agences gouvernementales américaines après avoir absorbé malgré lui toutes les informations confidentielles de la CIA. Non seulement l'avenir de la nation repose sur ses frêles épaules mais il doit en plus faire face aux mercenaires qui veulent s'emparer de lui coûte que coûte. La vie de Chuck se trouve donc bouleversée. De l'éternel adolescent égaré dans le monde adulte, il devient par la force des choses un agent secret sous couverture, épaulé par deux autres agents, la plantureuse Sarah Walker et le bourru John Casey. L'intérêt de Chuck, outre le postulat humoristique qu'elle propose, réside dans le double parcours initiatique du protagoniste. Au fur et à mesure de ses missions improvisées et toujours plus périlleuses, il développe une identité secrète, celle de Charles Carmichael, grâce à laquelle il va exprimer une facette radicalement différente de sa personnalité. Charles Carmichael est un agent secret téméraire que nul danger n'effraie. Il hausse le sourcil en lançant une pique sarcastique à ses ennemis tout en arborant un smoking sur-mesure. Et, peu à peu, cette identité souterraine va affecter la personnalité du vrai Chuck jusqu'à le rendre adulte. Par ces procédés, ce genre d'antihéros déclenche la compassion et la sympathie du spectateur. Chuck n'a pas l'allure et les pré-requis du héros et 
pourtant il va en devenir un. On se prend à s'attacher à ce personnage qui, dans sa maladresse et ses faille,s nous montre qu'il est avant tout humain.

\section{Les antihéros machiavéliques}

Un plus grand nombre d'antihéros aspirent, en apparence tout du moins, à sauver le " souverain bien » par tous les stratagèmes possibles, illustrant clairement l'idée que « la fin justifie les moyens ». Nous les nommerons "antihéros machiavéliques ». Ces personnages sont, au sens de John Stuart Mill ${ }^{17}$, des utilitaristes : ils considèrent la valeur morale d'une action uniquement en fonction de ses conséquences. Ainsi, si la finalité d'une action est de réparer une grave injustice, de prévenir une attaque terroriste ou bien de sauver un patient souffrant d'une maladie rare, les moyens pour y parvenir importent peu. Dans tous les cas, on cherche à atteindre un but moral. Cela suppose que l'antihéros machiavélique commet des entorses à la déontologie, à la justice et même à la morale tant qu'elles contribuent au bien du plus grand nombre. Nous pouvons néanmoins observer différents degrés d'utilitarisme parmi ces antihéros, selon leur propre personnalité.

Jack Bauer apparaît bien comme un digne descendant du Prince machiavélien. Individualiste forcené, Bauer décide par luimême et agit selon ses instincts, le plus souvent convaincu qu'il a raison ${ }^{18}$. Il n'hésite alors pas à torturer et à tuer si cela lui permet de réussir sa mission. En faisant cela, Bauer illustre le "principe d'agrégation » qui régit la pensée utilitariste puisqu'il envisage toujours ses actions à l'aune du bien-être du plus grand nombre, quitte à sacrifier une minorité19. L'utilitarisme peut ainsi recommander de faire du mal si cela peut éviter un mal encore plus grand. Au cours de la saison 2, il n'hésitera pas à exécuter de sang froid Ryan Chapelle, l'un de ses supérieurs à la Cellule Anti-terroriste, pour satisfaire les exigences de terroristes et ainsi éviter l'explosion d'une bombe nucléaire en plein Los Angeles. En tuant Chapelle, il permet la survie de millions de personnes, ce qui rend son crime finalement moral aux yeux des utilitaristes.

\footnotetext{
17 John Stuart Mill, L'Utilitarisme, Paris, éditions Flammarion, 2008.

${ }^{18}$ De toute façon, même en cas de doute, il fait toujours prévaloir son avis tant il est défiant vis-à-vis des ordres hiérarchiques.

19 Abordant ce même «principe d'agrégation » (ou maximisation du bonheur) dans son ouvrage, Philosophie en séries (Ellipses, 2009), Thibaut de Saint Maurice évoque un autre exemple patent dans 24 heures chrono : " quand l'agent Edmunds se retrouve attaché à une bombe bactériologique et que Jack décide de lui couper la main, pour évacuer la bombe, il fait exactement ce qu'il fallait faire, son choix est le bon, au sens où en termes de proportion de bonheur, la vie sauvée des civils aux alentours, est plus importante que la perte d'un de ses membres, pour un agent fédéral » p.21.
} 
Le sacrifice apparait d'ailleurs comme l'un des piliers de la série. Tout au long de ses trépidantes journées, il est prêt à tout sacrifier, famille, amis ou encore victimes innocentes jusqu'à prendre lui-même la place de la victime sacrificielle (il y laissera momentanément la vie à deux reprises) pour couper court aux crises inextricables dans lesquelles il se débat ${ }^{20}$. Toutefois, ces sacrifices sont rarement mis au service d'une raison d'Etat. Si Bauer est prêt à en être le martyr, celle-ci ne vaut jamais que l'on sacrifie qui que ce soit d'autre. D'autant moins que les autorités publiques vont révéler, tout au long des huit journées, des défaillances qui font naître chez lui une grande défiance vis-à-vis de tout ce qui incarne la puissance publique. L'exécution de Chapelle, qui en est le représentant, en constitue d'ailleurs une illustration à l'instar de sa désobéissance récurrente à sa hiérarchie. Sa dernière journée, durant laquelle il tente d'empêcher un accord international, confirme également que notre protagoniste a une tendance évidente à ne jamais vouloir se soumettre à la raison d'Etat. $\mathrm{Au}$ fond, Bauer cherche, en priorité, à éviter la contamination générale de la société par la violence et à restaurer les conditions d'une société en paix. S'il est prêt à sacrifier des vies, dont la sienne, c'est toujours pour sauver les fondements d'un vivre-ensemble, pour maintenir les conditions d'une vie pacifiée au sein de son monde ${ }^{21}$. Mais, en réalité, cette posture quasi-christique ${ }^{22}$ ne sert qu'à cacher le fait que, derrière des intentions en apparence altruistes, Jack Bauer n'est pas dénué de considérations égoïstes comme il le révèle dans Rédemption, le téléfilm servant de prologue à la $7^{\mathrm{e}}$ journée («Maybe I thought if I saved them, I'd save myself ${ }^{23}$.»). En définitive, si Bauer utilise tous les moyens pour servir l'intérêt général, c'est aussi parce qu'il recherche son intérêt particulier. On peut d'ailleurs douter de la réalité de ses propres sacrifices. Car, après le meurtre originel de son épouse Terri, notre personnage a-t-il vraiment encore quelque chose à perdre ? Les sept saisons suivantes n'auront de cesse de nous le montrer sous l'angle d'un être dépressif qui, après un passage par la drogue, élabore des plans beaucoup plus suicidaires que véritablement altruistes.

\footnotetext{
${ }^{20}$ Le sacrifice de soi apparaît d'ailleurs comme l'un des traits communs à nombre d'antihéros Ainsi, tant qu'il apparaît protecteur de ses équipiers, de sa famille voire de son quartier, Mackey, malgré ses méthodes, reste un antihéros. Dès lors qu'il sacrifie Ronnie, son dernier coéquipier encore vivant (7.13), pour sauver sa propre tête, il quitte ce statut. En n'étant pas capable de se sacrifier pour l'autre, il n'est alors plus au-dessus du Bien et du Mal, il bascule définitivement du côté du héros négatif, du côté du Mal.

${ }_{21}$ On rejoint l'analyse du sacrifice par René Girard pour qui l'ordre du monde repose précisément sur cette violence sacrificielle. La violence et le sacré, Paris, Hachette littérature, 1998.

${ }^{22}$ Ce qui est parfaitement illustré, au début de la 6 ème journée, par le retour sur le territoire américain d'un Bauer barbu et amaigri, attendu comme le Messie (il est le dernier espoir de sauver le monde) et prêt pour un nouveau sacrifice sur l'autel de la raison d'Etat

23 « Peut-être que je pensais qu'en les sauvant, je me sauverais moi-même».
} 
Ainsi, pour nos antihéros machiavéliques, l'action est amorale car elle n'est pas vraiment faite pour le bien-être de l'autre mais davantage pour son profit personnel. Dans Damages, le téléspectateur fait connaissance avec Patty Hewes, une avocate de renom prête à tout ce qui est nécessaire pour remporter son affaire, ne se souciant guère des dégâts collatéraux. Terriblement manipulatrice, Patty utilise aussi bien ses associés que ses clients, n'hésitant pas à mettre leur vie en danger ou à leur mentir effrontément afin de faire plier les multinationales auxquelles elle s'attaque. Mais derrière ses combats apparait toujours une justification morale. Dans chacune de ses affaires, elle représente et défend des personnes abusées par des magnats véreux et cupides. Elle ne fait pas cela pour l'argent - jamais elle ne demande de rémunération préalable. Mais, au final, on se rend compte que ces « croisades » contre de puissants groupes financiers ne vont pas être uniquement menées pour le bien des victimes. L'acharnement qu'elle démontre par exemple envers Arthur Frobisher, milliardaire sans scrupule qu'elle va ruiner et détruire au cours de la saison 1, prouve qu'elle cherche surtout à satisfaire un égo démesuré ou à prendre une revanche sur un passé que l'on découvrira par la suite. Si Patty donne l'illusion de vouloir protéger le bien commun, c'est bien pour défendre ses propres intérêts.

Le cas de Gregory House dans House MD est similaire. Ce dernier est un médecin aussi talentueux que misanthrope. Ayant développé une forte dépendance à la vicodine depuis son infarcissement à la jambe qui le contraint à marcher avec une canne, il officie à l'hôpital de Princeton-Plainsboro où il soigne des patients atteints de maladies rares. Le paradoxe est ici saisissant : comment un homme qui n'aime pas les autres peut-il être un très grand médecin ? Souvent comparé à juste titre à Sherlock Holmes 24 , House est en fait plus intéressé par l'énigme que représente la maladie que par le bienêtre du patient. Seule cette énigme semble l'animer : pour lui, la quête du bon diagnostic est un jeu pour lequel il est très doué puisqu'il sauve presque immanquablement tous ses patients. La guérison découle d'un bon nombre de manquements à l'éthique et à la morale. Mais encore une fois, en tant qu'utilitariste, House revendique que la fin justifie les moyens. Afin de soigner ses patients, atteints de maladies souvent graves et à un stade avancé, il n'hésite pas, par exemple, à ordonner aux jeunes internes qui le secondent d'entrer chez eux par effraction pour trouver d'éventuelles causes de la maladie. Si ses méthodes sont condamnables d'un point de vue moral - il peut aller jusqu'à

24 Au-delà de leur nom semblable, Holmes et House ont beaucoup en commun, de leur méthode d'investigation à leur fidèle compagnon, respectivement Watson et Wilson, sans oublier leur addiction à la drogue, cocaïne pour le premier, vicodine pour le second. On pourra se référer à l'article de Jerold $\mathrm{J}$. Abrams intitulé « La logique de l'hypothèse chez Sherlock Holmes et dans Dr. House " in H. Jacoby, dir., Dr House, les secrets d'un antihéros, Paris, Music Books, 2010. 
administrer un médicament contre-indiqué à un patient pour valider l'une de ses hypothèses - et s'il commet parfois des erreurs, il finit par œuvrer pour le bien du patient et parvient généralement à le soigner. Mais son intention n'est pas vraiment là et derrière cette recherche de résultat, il vise autre chose qui pourrait être la satisfaction de son narcissisme. D'ailleurs, les seuls patients auxquels House est réellement attentif sont ceux en qui il se reconnaît. C'est ainsi que dans l'épisode «Demi-prodige » (3.15), il témoigne une empathie qu'on ne lui connaissait pas pour Patrick, un patient avec qui il partage le plaisir de jouer du piano.

Si House et Hewes parviennent à faire croire qu'ils font prévaloir la finalité éthique d'agissements si peu déontologiques sur leurs intérêts personnels, Vic Mackey ne s'embarrasse pas d'une telle hypocrisie. Avec lui, nous arrivons à l'autre extrémité de notre nuancier avec un antihéros unique, à la marge de la philosophie utilitariste. Un personnage qui joue tellement sur les frontières du Bien et du Mal que la question de son statut - antihéros ou vrai méchant ? - se pose dès la première scène où il assassine Terry Crowley, l'un de ses coéquipiers susceptible de gêner ses combines. Mackey est agent de terrain dans les bas-fonds de Los Angeles, là où drogue et crime règnent en maîtres. Avec sa "strike team », unité spéciale antigangs musclée, il tente de remettre de l'ordre ou - à défaut - de contenir la violence dans ces quartiers sensibles. Toutefois, cette lutte contre la criminalité s'opère par des méthodes délictueuses si bien que Mackey devient vite un caïd local au même titre qu'un chef de gang, et tout aussi craint. Néanmoins, il finit toujours par arrêter les criminels ou les tuer quand ils mettent en péril son trafic personnel. Pour lui aussi, la fin justifie les moyens mais il semble, dans les premières saisons en tout cas, être habité par un désir de rendre les rues de Farmington plus sûres. C'est en ce sens qu'on peut lui accorder le statut d'antihéros. La réalité du terrain l'a amené à penser que pour lutter efficacement contre la délinquance, il fallait passer outre les considérations morales. Mais au fil des saisons, Vic Mackey va être rattrapé par le désordre, voire le chaos, qu'il crée dans son entourage pour maintenir l'ordre de sa propre vie. Figure autopoiétique 25 par excellence, constamment dans l'urgence d'avoir à réagir aux conséquences de ses actes, il est confronté à un effet «boule de neige » de plus en plus délicat à contenir. La dernière saison témoignera du point critique atteint par Vic qui, après avoir conduit plus ou moins directement à leur perte Curtis et Shane, les deux premiers membres de son équipe, se charge d'envoyer Ronnie en prison à sa place. La boucle est bouclée, de l'abject meurtre originel à l'ignominie finale en passant par le sacrifice de l'innocent Guardo (3.6), l'autre «Quatuor de Los Angeles » (après celui

${ }^{25}$ L'autopoï̀se est la propriété d'un système qui consiste à maintenir son ordre en créant du désordre dans son environnement. 
d'Ellroy) est décimé et Mackey bascule du statut d'antihéros vers celui de véritable criminel sans foi, ni loi, ni cœur.

\section{Les antihéros maudits}

A l'inverse, un tueur en série comme Dexter Morgan a su créer une connivence avec le public. On en viendrait presque à oublier que la nuit, cet enquêteur de Miami découpe ses victimes en petits morceaux. Mari et beau père exemplaire, collègue apprécié de tous, il apparaît comme un homme sympathique parfaitement intégré dans la vie sociale. Tout ce que n'est pas Vic Mackey. Mais la différence majeure avec ce dernier - et qui va jouer sur notre compréhension du personnage - tient finalement au fait que son passé nous est conté.

Nous savons dès la première saison pourquoi Dexter est un tueur. Alors qu'il n'était qu'un enfant, il a assisté au meurtre abominable de sa mère et est resté assis dans son sang des jours durant jusqu'à ce que la police le retrouve enfin. Cet événement aura laissé des séquelles sur la personnalité du petit Dexter qui va développer un irrépressible besoin de tuer. Grâce à son père adoptif, il parvient à le canaliser et à le diriger. En effet, Dexter ne tue que de vrais criminels qui ont échappé aux mailles de la justice, après s'être au préalable assuré de leur culpabilité. Ce rituel immuable qui lui permet de ne presque jamais faire d'erreur est rendu possible par son métier de spécialiste en morpho-analyse de taches de sang au sein de la police criminelle de Miami. Dexter s'est ainsi donné l'illusion de respecter une éthique du meurtre: en ne tuant que des criminels, il lutterait à sa manière contre la récidive et pour le bien de tous. Si son passé n'excuse aucunement l'atrocité des meurtres qu'il commet, il permet de comprendre Dexter et de lui donner des circonstances atténuantes. Si, nous l'avons vu, tous les antihéros ont leur " part maudite », Dexter est un antihéros avant tout maudit au sens où cette malédiction est constitutive, qu'elle l'a créé et qu'elle explique tout son quotidien.

Dexter Morgan apparaît ici comme un antihéros particulièrement original, probablement l'un des premiers antihéros postmodernes des séries télévisées se distinguant, par exemple, d'un personnage comme Jack Bauer. Ce dernier se situe en effet dans la dramaturgie caractéristique d'une modernité obsédée par le futur, l'élaboration d'un projet et la mise en place d'actions pour le réaliser. L'agent de la CTU apparaît mû par un projet, une perspective éthique visant à vivre avec et pour les autres au sein d'institutions protectrices. Il est, de fait, constamment dans la praxis en échafaudant des plans rationnels pour trouver sans cesse des solutions à la cascade de difficultés qu'il doit affronter. Rien de tel chez Dexter qui semble accepter l'existence pour ce qu'elle est, sans autre volonté que celle de vivre au présent qui constitue les fondements de la société 
postmoderne. L'individu n'y est plus vraiment acteur mais davantage manipulé par des forces qui le dépassent. Dexter est un antihéros tragique entièrement déterminé par un sombre Destin qu'il ne cherche pas à contrarier mais simplement à accommoder grâce à un code appris par son père, Harry ( «never get caught, never kill an innocent $\left.{ }^{26} »\right)$. Tout au long des cinq premières saisons, il lui sera d'ailleurs rappelé qu'il ne peut échapper à ce destin tragique à travers la perte d'êtres qui auraient pu lui faire retrouver un supplément d'humanité et lui faire reprendre en mains le cours de son existence. Qu'il s'agisse de Rita, son épouse qui s'éteindra dans le bain de son propre sang à la fin de la saison 4 (4.12), ou de Lumen, sa camarade de jeu de la 5ème saison, qui avait pourtant accepté son « Passager Noir », toutes l'abandonnent à son triste sort. Dans la société postmoderne, pour Dexter pas plus que pour d'autres, il n'existe ni « grand soir » ni " lendemains qui chantent ». Loin de préparer un avenir plus faste, il ne fait que répéter le mythe originel sanglant à travers un ritualisme qui signe lui-aussi sa postmodernité ${ }^{27}$.

\section{Le héros existe-t-il encore ?}

Notre panorama des antihéros des séries dramatiques contemporaines visait à montrer le changement radical des attentes spectatorielles. Un désir de réalisme a prospéré depuis dix ans sous l'impulsion, comme nous l'avons mentionné, de l'avènement de la téléréalité et des attentats du 11 septembre 2001 qui ont redéfini le paysage télévisuel. Les antihéros sont devenus une réponse inattendue à un climat d'anxiété oppressant. Alors que l'on aurait pu penser que les héros infaillibles, fières figures patriotiques, se multiplieraient, il en fut tout autrement. Le téléspectateur semble alors s'identifier à des personnages plus sombres et ambigus qui, par des chemins détournés, vont, par exemple, réussir là où les agences gouvernementales ont échoué (prévenir la menace terroriste, arrêter un coupable...). Il oublie le temps d'un épisode ce qui est arrivé grâce à une réécriture de l'histoire, la mise en place d'une uchronie ${ }^{28}$, qui va lui servir d'opium. Mais aussitôt l'illusion achevée, le retour à la réalité est d'autant plus douloureux. Les antihéros demeurent très présents aujourd'hui sur nos écrans ; pour autant, le héros traditionnel a-t-il totalement disparu ?

$\mathrm{Au}$ regard des séries contemporaines, il semble que ce dernier existe toujours mais il est lui aussi affecté par le climat de son époque.

${ }^{26}$ "Ne jamais se faire prendre, ne jamais tuer un innocent. »

${ }_{27}$ M. Maffesoli, L'instant éternel, Paris, La table ronde, 2003.

${ }^{28}$ Par uchronie, on désigne la réécriture, la réinvention, de l'histoire. On imagine ce qui aurait $p u$ arriver en changeant un événement fondateur. Par exemple, lorsque Jack Bauer met hors d'état de nuire des terroristes présents sur le territoire américain, il opère une dénégation du 11 septembre en les empêchant de passer à l'acte. Ainsi, il permet virtuellement à l'Amérique d'échapper à son histoire. 
Devenu plus ambigu et sombre, le héros d'aujourd'hui s'en est trouvé humanisé. Une série comme Heroes (NBC, 2006-2010) est un bon exemple de cette tendance car, en dépit de leurs pouvoirs, les personnages principaux ne sont pas des super héros mais bien des héros. La différence tient au fait que l'on insiste ici sur un héroïsme qui ne résulte pas des seules capacités extraordinaires mais aussi et surtout de qualités humaines de courage et de bravoure. Et la série s'attardera particulièrement sur l'intimité des personnages en montrant leurs failles et leurs doutes.

Dans d'autres séries d'action comme Human Target (Fox, 2010-2011), on retrouve davantage la figure du héros canonique au sens où la psychologie du protagoniste est un enjeu secondaire : la mission prévaut. C'est un traitement que l'on retrouve également dans des séries procédurales comme Les Experts (CBS, 2000-) ou Esprits Criminels (CBS, 2005-). Mentionnons pour finir The Good Wife (CBS, 2009-), série dans laquelle Alicia Florrick incarne une vraie héroïne au sens moderne. C'est une femme bafouée qui va faire face au jugement des autres pour se construire en tant que femme indépendante en devenant une avocate réputée tout en subissant une vie privée difficile. Avec elle, la notion d'héroïsme est fondamentalement humanisée. Le héros est désormais sur le même registre que le commun des mortels, il est désacralisé et donc accessible. Les enjeux n'ont ici rien d'extraordinaire, Alicia ne va pas sauver le monde. Cette dernière est juste une femme comme les autres, une héroïne du quotidien. Et c'est finalement ce quotidien et cette proximité qui vont permettre au téléspectateur d'adhérer à The Good Wife. Car c'est bien en exerçant son métier de femme qu'Alicia Florrick révèle sa capacité à faire preuve d'héroïsme.

Devant ces protagonistes si humains, nous avons à faire à des figures qui ne sont ni des héros ni des antihéros. Il faut alors envisager que l'héroïsme puisse emprunter une troisième modalité qui pourrait correspondre à ce «eroe a rovescio » qu'évoque Primo Lévi ${ }^{29}$. Ce «héros à rebours » n'est ni un héros (cet être supra-humain), ni un héros en négatif, capable de se fourvoyer dans des chemins peu recommandés, comme l'est l'antihéros. Pendant que le héros, être extraordinaire, descend vers le monde réel pour y dévoiler toutes ses qualités, notre «héros à rebours » emprunterait le cheminement inverse : il partirait de l'humain et monterait vers l'héroïsme.

${ }^{29}$ Primo Levi, La recherche des racines, Turin, Einaudi, 1998. 